\title{
Embarazo abdominal: Caso clínico y revisión
}

\author{
Jaime Alberto Messa Acosta*; Antonio Solorza López**
}

RESUMEN: El embarazo abdominal es una forma rara de embarazo ectópico con alta morbi-mortalidad materno fetal. Un manejo quirúrgico apropiado e individualizado de pacientes embarazadas con este problema, puede ayudar a prevenir complicaciones y mortalidad. Reportamos un caso con revisión de la literatura.

PALABRAS CLAVES: Embarazo abdominal, embarazo ectópico.

SUMMARY: Abdominal pregnancy is a rare from of ectopic pregnancy, with high degree of maternal and fetal mortality and morbidity. Appropriate and individualized operative management of pregnant patients with this problem, can avert many of its complications and associated mortality. We report a case and review of the literature.

KEY WORDS: Abdominal pregnancy, ectopic pregnancy.

\section{Caso clínico}

M.F. HC No. 404563 Edad: 15 años procedente de área rural. Remitida con diagnóstico de feto muerto y ecografía que demuestra feto en presentación podálica, sin latido cardíaco ni movimientos fetales. Ingresa el 14 de Nov-1995 con los siguientes datos clínicos:

Fecha de última menstruación fiable del 25 Jun-94 (16 meses) y fecha probable de parto para el $1^{\circ}$ abril-95. En noviembre-94 se realizó test de embarazo el cual es positivo. En abril-95 deja de percibir movimientos fetales y desde marzo-95 hasta el ingreso presenta sangrado vaginal tipo menstrual cíclico $\mathrm{c} / 30$ días.

Antecedentes: Menarca $=11$ años Nunca planificación, nunca citologías ciclos previos: irregulares inicio de actividad sexual a los 12 años.

Examen clínico: Signos vitales normales. Altura uterina $=24 \mathrm{cms}$. Se palpan partes fetales sin movimientos ni fetocardia. Escaso sangrado vaginal con orificio cervical central y cerrado. Al tacto bimanual masa izquierda que no hace cuerpo con el útero de 10x12 cms. La movilización del feto se hace libre en la cavidad abdominal sin movilización del cérvix. Se toma una Ecografía que reporta feto intrauterino único muerto cefálico, biometría para 26 semanas, índice de líquido amniótico de 15 y cabalgamiento a de huesos craneales. Ante la sospecha clínica de embarazo abdominal se solicita una histerografía la cual con aplicación de $20 \mathrm{cc}$ de medio de contraste se visualiza útero reducido de tamaño de forma irregular con visualización de sólo trompa derecha e imagen de cabeza fetal de manera extrínseca al útero. (Figura 1). Se toman hemograma, plaquetas, uroanálisis,

\footnotetext{
* Residente III año. Universidad del Cauca Docente. Universidad del Cauca
}

\section{Figura 1 \\ HISTEROGRAFIA DONDE SE OBSERVA LLENAMIENTO DEL MEDIO DE CONTRASTE EN LA CAVIDAD UTERINA CON LA CALOTA FETAL EXTRINSECAMENTE}

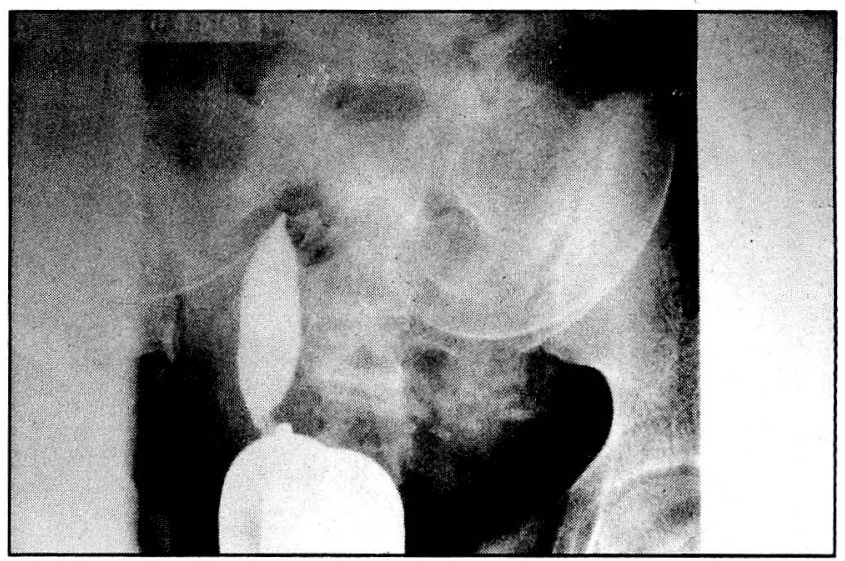

serología, tiempos de coagulación, fibrinógeno y productos de degradación de fibrina los cuales son normales. Se cruza y reservan 3 unidades de sangre y se lleva la paciente a laparotomía encontrando saco gestacional libre en cavidad abdominal y placenta adherida al epiplón (Figura 2). Se realiza excerésis del saco con omentectomía parcial logrando extraer feto (Figura 3) y la totalidad de la placenta. Presenta además útero con cuerno izquierdo rudimentario y alteración en la morfología tubaria izquierda. (Figura 4). La recuperación de la paciente en el post operatorio es satisfactoria. 
Figura 2

SE OBSERVA EL SACO AMNIOTICO INDEMNE CON LA PLACENTA LA CUAL HA SIDO PARCIALMENTE RESECADA DEL EPIPLON

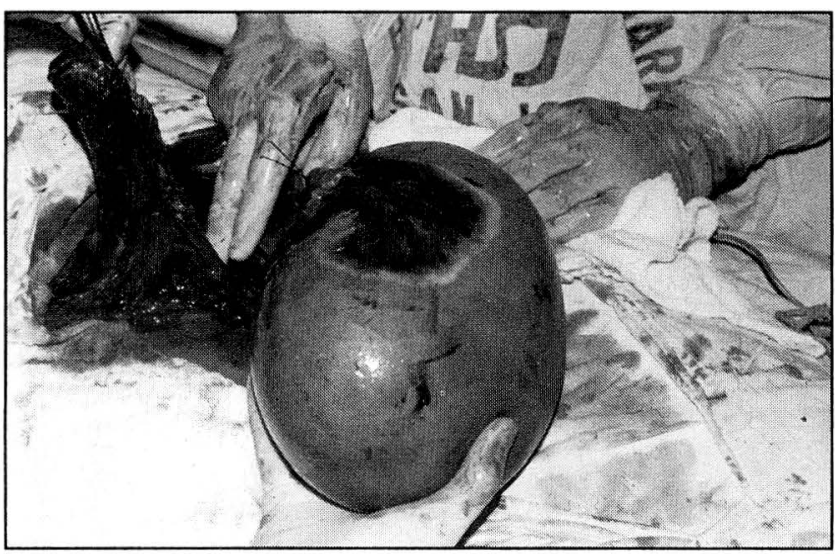

Figura 3

AL SECCIONAR EL SACO AMNIOTICO SE APRECIA FETO MUERTO MACERADO. NOTESE EL ELEVADO GROSOR DE LAS MEMBRANAS FETALES

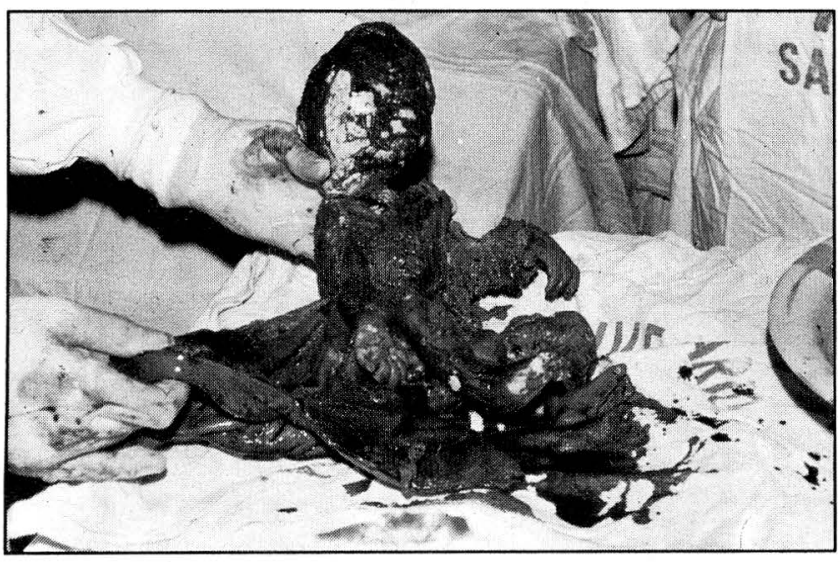

\section{EMBARAZO ABDOMINAL}

\section{Definición}

Variante rara de embarazo ectópico el cual se desarrolla en la cavidad abdominal con implantación placentaria en cualquiera de sus estructuras y crecimiento fetal.

\section{Epidemiología}

Las estadísticas se ven influenciadas por la incidencia de embarazos ectópicos en la población, uso de técnicas de reproducción asistida y capacidad de control prenatal que permitan diagnósticos precoces (1). El riesgo de morir por un embarazo abdominal es 7.7 veces mayor que un embarazo tubario y 90 veces mayor que el embarazo intrauterino normal (2). La mortalidad materna es de 0.5 -
Figura 4

\section{ANOMALIA UTERINA CON FORMACION DE UN CUERNO RUDIMENTARIO. AMBOS OVARIOS SON MACROSCOPICAMENTE NORMALES}

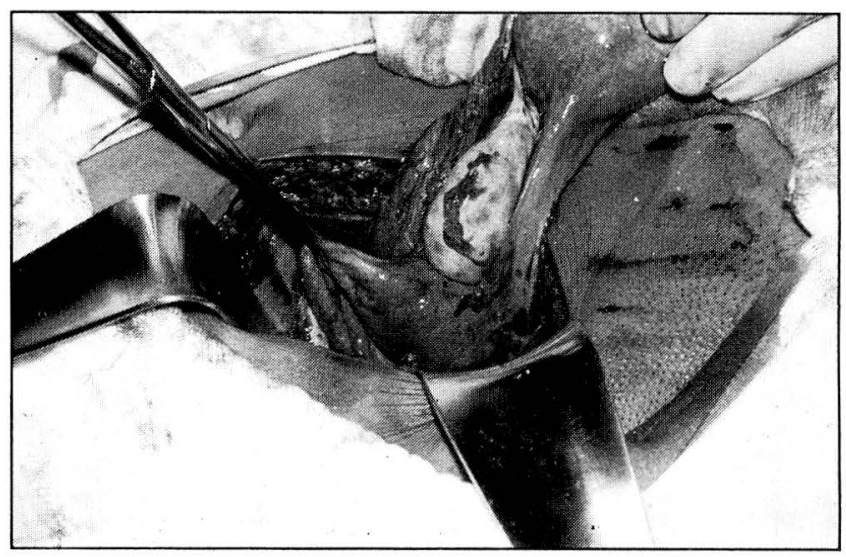

$18 \%$ y la mortalidad fetal de $40-95 \%(3-5)$. La incidencia es de 10,9-20,6 por cada 100.000 Nacim. y 9,2-14.5 por cada 1.000 embarazos ectópicos (3-4) en Colombia se han descrito varios casos (6).

Casos raros de gestación gemelar abdominal (7), asociados con mola parcial (8), embarazos heterotópicos con ambos recién nacidos vivos (9-10), e incluso en paciente con antecedente de histerectomía (11) se han descrito en la literatura mundial.

\section{Factores de riesgo}

Similares al embarazo ectópico a saber son antecedente de infección pélvica-anomalías congénitasendometriosis-embarazo ectópico previo (3) -Cirugía uterina previa- Técnicas de reproducción asistida que incluyen fertilización invitro (12-13), e inducción de ovulación (13), Tuberculosis (1) etc.

\section{Fisiopatología}

Existen dos tipos de embarazo abdominal:

a. Primario: el cual la fertilización se realiza a nivel peritoneal con subsiguiente implantación. Este tipo es más raro. Para hacer el diagnóstico de embarazo abdominal primario debe cumplir con los siguientes criterios enunciados por Studdiford (14).: 1- Trompas sanas sin datos de nidación previa. 2- Ausencia de fístulas útero placentarias o ruptura uterina previa. 3- Producto adherido sólo a estructuras peritoneales.

b. Secundario: Es el más frecuente y se presenta por lo general secundario a ruptura de un embarazo en la trompa con implantación posterior o a un aborto tubario (15).

Diferenciar entre primario o secundario tiene poca significancia clínica pues el manejo es el mismo (3).

Niveles hormonales de Estrógenos, progesterona y gonadotropinas son similares a un embarazo normal (1, 16). La placenta puede mostrar actividad hormonal hasta 7 semanas postparto cuando es dejada in-situ (16). 


\section{Clínica}

No existe una sintomatología específica para esta entidad sin embargo el dolor abdominal inexplicable es el síntoma primordial como el dolor con los movimientos fetales (2-3). Los signos clínicos que se pueden encontrar son mala presentación fetal, ausencia de reactividad uterina y palpación fácil de partes fetales (1, 5 17-18).

Puede observarse incremento de alfa fetoproteína (2-3).

La ausencia de contracciones uterinas o ausencia de registro por un tocodinamómetro pueden ser sospechosas de embarazo abdominal $(5-6,19-20)$ pero puede haber registro de actividad si la placenta se localiza en la parte posterior al útero desplazándolo hacia la cavidad abdominal dando un test falso positivo (19).

\section{Diagnóstico}

Sólo $10-30 \%$ de las pacientes son llevadas a cirugía con un diagnóstico pre operatorio de embarazo abdominal $(4,17)$ esto se debe básicamente a la falta de familiaridad del obstetra con este tipo de casos debido a lo poco frecuente de su presentación $(4,21)$.

Sin embargo se han descrito varios elementos que puedan llevar a sospechar o a diagnosticar esta patología.

Radiografía Simple de abdomen: Todos los hallazgos son inespecíficos como pequeñas partes fetales inmediatamente sobre la columna materna, feto alto en el abdomen, sombra uterina separada del feto, feto en mala presentación (18) y sombras intestinales limitando con partes fetales (17).

Ecografía: Sólo logra demostrar el $60 \%$ de los casos (15) y en ocasiones el ultrasonido para diagnóstico puede ser desorientador (22), siendo muy difícil su identificación en el primer trimestre del embarazo (23). Los hallazgos ecográficos más frecuentes son partes fetales separadas del útero, falta de visualización de la pared uterina entre el feto y la vejiga, mayor aproximación entre partes fetales y pared abdominal materna, feto en posición excéntrica y dificultad para una adecuada visualización placentaria $(5,17-18,24)$.

Escanografia: Puede usarse no sólo para evaluar la gestación abdominal sino para hacer un seguimiento placentario posterior a la extracción del feto siendo de elección cuando se encuentra asociado con feto muerto $(2,24)$.

Resonancia Magnética Nuclear: Tiene la ventaja sobre la escanografía de que no se están usando radiaciones ionizantes con una excelente visualización placentaria (3, 25-27).

Histerografía: Hace el diagnóstico en todos los casos al observar llenado del medio de contraste en la cavidad uterina con partes fetales fuera de la misma sin embargo no se debe realizar ante sospecha de embarazo intrauterino viable (3).

\section{Complicaciones}

A. Fetales: Son numerosas las complicaciones fetales principalmente por alteraciones del intercambio feto materno con fragilidad en el sitio de implantación y disrupción vascular lo que conllevaría a retardo en el crecimiento con marcado oligoamnios (2) y alteraciones por el mismo como son hipoplasia pulmonar y alteraciones en las extremidades (15).

El uso del Doppler puede servir para evaluar la circulación fatal en el embarazo abdominal (28) mostrando ondas similares al embarazo normal (1).

Se observan anomalías fetales que incluyen malformaciones y deformidades con una tasa combinada de 20 $40 \%(17,29)$.

B. Maternas: La principal complicación materna la constituye el sangrado sobre todo en el momento de la cirugía al intentar remoción del tejido placentario. Sin embargo el hecho de dejar la placenta en la cavidad abdominal también presenta graves complicaciones dentro de las que se incluyen hemorragia secundaria-hipofibrinogenemia-pre eclampsia/eclampsia (30)-perforación intestinal-obstrucción intestinal-reacciones febriles-septicemia-peritonitis-formación de abscesos (2) y posibilidad remota del desarrollo de un coriocarcinoma (31).

\section{Manejo}

En casos aislados la cirugía puede ser postpuesta para permitir la maduración pulmonar fetal $(3,15)$. Los criterios para este manejo conservador son: 1- Que el feto estẻ vivo y tenga una edad gestacional avanzada 2 - Ausencia de malformaciones congénitas mayores 3-Presencia de bienestar fetal 4- Implantación placentaria lejos del hígado o bazo 5- Personal disponible adecuado (2).

Manejo de la placenta: Se han propuesto varios esquemas terapéuticos (3) que dependen de la localización placentaria, la disponibilidad de sangre y el estado de viabilidad placentaria $(18,32)$; estos esquemas son:

1. Evitar la manipulación placentaria dejando la placenta en su lugar y sin ningún manejo posterior.

2 . Remover órganos pélvicos sobre la cual esté implantada, es el caso de la histerectomía.

3. Dejar la placenta en su lugar y luego administrar Metrotexate.

Por lo tanto si la placenta es quirúrgicamente removible sin acarrear un sangrado masivo como es el caso de implantaciones en el epiplón ésta podría resecarse sin embargo esto no se cumple en la mayoría de los casos por lo que el tratamiento preferible sería dejar la placenta en la cavidad abdominal sin ningún tipo de manejo posterior, pues se ha observado que el uso de metrotexate conlleva a una destrucción placentaria muy acelerada con acúmulo de tejido necrótico en cantidades mayores lo que favorecería la infección (24).

Ante sangrado masivo se ha utilizado embolización selectiva por cateterización de la arteria femoral con émbolos de gelfoam (24). La extracción fetal una vez se logra la incisión del saco amniótico debe hacerse muy cuidadosa, se ha descrito hemorragia retroplacentaria inmediatamente posterior a la remoción del feto (17).

La placenta puede ser extirpada cuando exista cese de la actividad hormonal de HCG (33) o si el feto lleva más de cuatro semanas de muerto (2).

Si la placenta es dejada en su lugar, algunos autores recomiendan monitorizarla con ecografía y gonadotropinas en el post operatorio para observar involución (24). 
No se recomienda dejar dren en la pelvis luego de cirugía (3) esto favorece un foco de infección (17).

En gestaciones muy precoces, autores han utilizado manejo laparoscópico (34) sin embargo son necesarios más estudios al respecto.
El Metotrexate sólo pudiera ser útil en casos de obstrucción intestinal o en casos de niveles progresivamente crecientes de b-HCG en el post parto (2).

\section{BIBLIOGRAFIA}

1. Cunningham FG., Mc Donald PC., Leveno KJ., Williams Obstetric 19th Edition Norwalk. Appleton-Lange 1993; 710-713.

2. Martin JN., Jr. McCaul JF. Tratamiento de urgencia del embarazo abdominal. Clin. Obstet. Ginecol. 1990; 3: 427-435.

3. Martin JN., Jr. Sessums JK., Martin RW et al. Abdominal pregnancy: Current concepts of Management. Obstet. Gynecol. 1988; 71:549-557.

4. Atrash HK., Friede A., Hogue C. Abdominal pregnancy in the United States-Frequency and maternal mortality. Obstet. Gynecol. 1987; 69: 333-337.

5. Delke I., Pérez N., Trancer ML. Abdominal Pregnancy: Review of Current Management and Addition of 10 Cases. 1982; 60: 200-203.

6. Mariño MA., Heredia MM. Embarazo abdominal. Rev. Col. Obstet. Ginecol. 1992; 43: 70-71.

7. Stovall TG., Elkins TE. Twin abdominal pregnancy: A case report. J. Reprod. Med. 1985; 30: 784.

8. Cordero DR., Adra A., Yasin S. Intraligamentary pregnancy. Obstet. Gynecol. Surv. 1994; 49: 206-209.

9. Crabtree KE., Collet B., Kilpatrick SJ. Puerperal presentation of a living abdominal pregnancy. Obstet. Gynecol. 1994; 84: 646-648.

10. Bassil SJ., Pouly L., Canis $M$ et al. Advanced heterotopic pregnancy after in vitro fertilization and embryo transfer, with survival of both the babies and mother. Hum. Reprod. 1991; 146: 515-525.

11. Nehra PC., Loginsky SJ. Pregnancy after vaginal hysterectomy. Obstet. Gynecol. 1984; 64: 735.

12. Ferland RJ., Chadwick DA., O'Brien JA et al. An ectopic pregnancy in the upper retroperitoneum following in vitro fertilization and embryo transfer. Obstet. Gynecol. 1991; 78: 544-546.

13. Cheng WF., Ho NH., Yang YS et al. Abdominal pregnancy after gonadotropin superovulation and intrauterine insemination: A case report. Am. J. Obstet. Gynecol. 1994; 171: 1394-1395.

14. Studdiford WE. Primary peritoneal pregnancy. Am. J. Obstet. Gynecol. 1942; $44: 487$.

15. Jones HW., Wentz AC., Burnett LS. Textbook of gynecology. 11th edition Maryland Williams and Wilkins 1988; 439-440.

16. France JT., Jackson P. Maternal plasma and urinary hormone levels during and after a successful abdominal pregnancy. Br. J. Obstet. Gynecol. 1980; 87: 356-362.

17. Rahman MS., Al-Suleiman SA., Rahman J et al. Advanced abdominal pregnancy: Observations in 10 Cases. Obstet. Gynecol. 1982; 59: 366-372.
18. Strafford JC., Ragan WD. Abdominal pregnancy: Review of current Management. Obstet. Gynecol. 1977; 50: 548-552.

19. Orr JW., Huddleston JF., Knox GE et al. False negative oxytocin challenge test associated with abdominal pregnancy. Am. J. Obstet. Gynecol. 1979; 133: 108-110.

20. Leikin E., Randall HW Jr. Hydrocephalic fetus in an abdominal pregnancy. Obstet. Gynecol. 1987; 69: 498-500.

21. Angtuaco TL., Shah HR., Neal MR et al. Ultrasound evaluation of abdominal pregnancy. Crit. Rev. Diagn. Imaging. 1994; 35: 1-59.

22. Ali V., Saldana LR., Balat IY et al. Pitfalls in sonographic diagnosis of abdominal pregnancy. South. Med. J. 1981; 74: 477.

23. Graham MF. First trimester abdominal pregnancy. J. Clin. Ultrasound. $1977 ; 5: 321$.

24. Akhan O., Cekirge S., Senaati S et al. Sonographic diagnosis of an abdominal ectopic pregnancy. Am. J. Radiol. 1990; 155: 197.

25. Harris MB., Antgtuaco T., Frazier $\mathrm{CN}$ et al. Diagnosis of viable abdominal pregnancy by magnetic resonance imaging. Am. J. Obstet. Gynecol. 1988; 159: 150-151.

26. Spanta R., Roffman LE., Grissom TJ et al. Abdominal pregnancy. Magnetic resonance identification with ultrasonographic follow-up of placental involution. Am. J. Obstet. Gynecol. 1987; 157: 887-889.

27. Wagner A., Burchardt AJ. MR. Imaging in advanced abdominal pregnancy: A case report of fetal death. Acta-Radiol. 1995; 36: 193195.

28. Hage ML., Wall LL., Killam A. Expectant management of abdominal pregnancy. J. Reprod. Med. 1988; 33: 407.

29. Stevens SA. Malformations and deformations in abdominal pregnancy. Am. J. Med. Genet. 1993; 47: 1189-1195.

30. Piering WF., Garancis JG., Becker CG et al. Preeclampsia related to a functioning extrauterine placenta: Report of a case and 25-year follow up. Am. J. Kidney Dis. 1993; 21: 310-313.

31. Jackson RL. Pure malignancy of the trophoblast following primary abdominal pregnancy. Am. J. Obstet. Gynecol. 1960; 79: 1085.

32. Hreshchysshyn NM., Bogen B., Loughran CH. What is the actual present-day management of the placenta in late abdominal pregnancy? Analisis of 101 cases. Am. J. Obstet. Gynecol. 1961; 81: 320.

33. Thompson JD., Rock JA. Te Linde Ginecologia quirúrgica $7^{\circ}$ ed. Buenos Aires Panamericana 1993; 401-402.

34. Dover RW., Powell M. Management of a primary abdominal pregnancy. Am. J. Obstet. Gynecol. 1995; 172: 1603-1604. 